\title{
Skills covered within the Case Studies
}

\begin{tabular}{|c|c|c|c|c|c|c|c|c|}
\hline Skill Ref. & 1 & 2 & 3 & 4 & 5 & 6 & 7 & 8 \\
\hline $1 \mathrm{a}$ & & & & & $\mathrm{A}$ & $\mathrm{A}$ & A & \\
\hline $1 \mathrm{~b}$ & & & & & & & & \\
\hline $1 \mathrm{c}$ & & & & & & & & \\
\hline $1 \mathrm{~d}$ & & & & & & & & \\
\hline le & & & & & & & & \\
\hline If & & & & & & & & \\
\hline $1 \mathrm{~g}$ & $\mathrm{~A}$ & & & & & & $\mathrm{~A}$ & A \\
\hline $1 \mathrm{~h}$ & & & $\mathrm{~A}$ & & & & & \\
\hline $1 \mathrm{i}$ & $\mathrm{A}$ & $\mathrm{A}$ & & & & & & \\
\hline $1 \mathrm{j}$ & $\mathrm{A}$ & $\mathrm{A}$ & & $\mathrm{A}$ & & & $\mathrm{A}$ & $\mathrm{A}$ \\
\hline $1 \mathrm{k}$ & & & & $\mathrm{A}$ & & & & \\
\hline 11 & & & & $\mathrm{~A}$ & & & & \\
\hline $1 \mathrm{~m}$ & & & & $\mathrm{~A}$ & & & & A \\
\hline $\ln$ & $\mathrm{A}$ & $\mathrm{A}$ & $\mathrm{A}$ & & & & & $\mathrm{A}$ \\
\hline 10 & & & & & & & & \\
\hline $1 p$ & & & & & & & & \\
\hline $1 q$ & & & & & $\mathrm{~A}$ & & $\mathrm{~A}$ & \\
\hline $1 \mathrm{r}$ & & & & & & & & \\
\hline $1 \mathrm{~s}$ & & $\mathrm{~A}$ & $\mathrm{~A}$ & $\mathrm{~A}$ & & A & & \\
\hline $2 b$ & $\mathrm{~A}$ & & & & $\mathrm{~A}$ & & & \\
\hline $2 c$ & $\mathrm{~A}$ & & & & & & & \\
\hline $2 \mathrm{~d}$ & & & & & & & & \\
\hline $2 \mathrm{e}$ & $\mathrm{A}$ & $\mathrm{A}$ & & $\mathrm{A}$ & $\mathrm{A}$ & $\mathrm{A}$ & $\mathrm{A}$ & $\mathrm{A}$ \\
\hline $2 \mathrm{f}$ & $\mathrm{A}$ & & & $\mathrm{A}$ & & & & $\mathrm{A}$ \\
\hline $2 \mathrm{~g}$ & $\mathrm{~A}$ & & & & $\mathrm{~A}$ & & & $\mathrm{~A}$ \\
\hline $2 \mathrm{~h}$ & & & & & & & & \\
\hline $2 \mathrm{i}$ & $\mathrm{A}$ & & & & & & & \\
\hline $2 \mathrm{j}$ & & $\mathrm{A}$ & $\mathrm{A}$ & & $\mathrm{A}$ & & $\mathrm{A}$ & \\
\hline $3 a$ & $\mathrm{~A}$ & & $\mathrm{~A}$ & $\mathrm{~A}$ & & & & $\mathrm{~A}$ \\
\hline $3 b$ & $\mathrm{~A}$ & & $\mathrm{~A}$ & $\mathrm{~A}$ & & & & \\
\hline $3 c$ & & $\mathrm{~A}$ & $\mathrm{~A}$ & $\mathrm{~A}$ & $\mathrm{~A}$ & & $\mathrm{~A}$ & $\mathrm{~A}$ \\
\hline $3 \mathrm{~d}$ & & & $\mathrm{~A}$ & & $\mathrm{~A}$ & & & $\mathrm{~A}$ \\
\hline $3 e$ & & & & & & & & \\
\hline $3 \mathrm{f}$ & & & & & & & & \\
\hline $3 g$ & & & & $\mathrm{~A}$ & & $\mathrm{~A}$ & $\mathrm{~A}$ & \\
\hline $3 \mathrm{~h}$ & & & & & & & & $\mathrm{~A}$ \\
\hline $3 \mathrm{i}$ & & & $\mathrm{A}$ & & & & & \\
\hline $3 j$ & & & & & & & & \\
\hline $3 \mathrm{k}$ & & & & & & & & \\
\hline 31 & & $\mathrm{~A}$ & & & $\mathrm{~A}$ & $\mathrm{~A}$ & & \\
\hline $3 \mathrm{~m}$ & & & & & & & & \\
\hline $4 a$ & $\mathrm{~A}$ & & $\mathrm{~A}$ & & & $\mathrm{~A}$ & & \\
\hline $4 \mathrm{~b}$ & & & & & A & & $\mathrm{A}$ & \\
\hline $4 c$ & $\mathrm{~A}$ & $\mathrm{~A}$ & & $\mathrm{~A}$ & & & $\mathrm{~A}$ & $\mathrm{~A}$ \\
\hline $4 d$ & & $\mathrm{~A}$ & $\mathrm{~A}$ & & & & & \\
\hline $4 e$ & $\mathrm{~A}$ & A & & A & $\mathrm{A}$ & A & & \\
\hline $4 \mathrm{f}$ & & & & & & & & \\
\hline
\end{tabular}

\begin{tabular}{|c|c|c|c|c|c|c|c|c|}
\hline Skill Ref. & 9 & 10 & 11 & 12 & 13 & 14 & 15 & 16 \\
\hline la & & & A & & & & & \\
\hline $1 \mathrm{~b}$ & & $\mathrm{~A}$ & & & & & & \\
\hline \multicolumn{9}{|l|}{ lc } \\
\hline \multicolumn{9}{|l|}{$1 \mathrm{~d}$} \\
\hline le & $\mathrm{A}$ & & & & & & & \\
\hline \multicolumn{9}{|l|}{ If } \\
\hline $1 \mathrm{~g}$ & $\mathrm{~A}$ & & $\mathrm{~A}$ & & & $\mathrm{~A}$ & & $\mathrm{~A}$ \\
\hline $1 \mathrm{~h}$ & & & & $\mathrm{~A}$ & & & & \\
\hline li & & $\mathrm{A}$ & & & $\mathrm{A}$ & & & $\mathrm{A}$ \\
\hline $1 j$ & & & & $\mathrm{~A}$ & $\mathrm{~A}$ & & & \\
\hline \multicolumn{9}{|l|}{$1 \mathrm{k}$} \\
\hline \multicolumn{9}{|l|}{11} \\
\hline $1 \mathrm{~m}$ & $\mathrm{~A}$ & & & & & & $\mathrm{~A}$ & \\
\hline $\ln$ & & & & & $\mathrm{A}$ & & $\mathrm{A}$ & \\
\hline \multicolumn{9}{|l|}{10} \\
\hline $1 p$ & & & $\mathrm{~A}$ & & & $\mathrm{~A}$ & $\mathrm{~A}$ & \\
\hline $1 \mathrm{q}$ & & & $\mathrm{A}$ & & & & $\mathrm{A}$ & $\mathrm{A}$ \\
\hline \multicolumn{9}{|l|}{$1 \mathrm{r}$} \\
\hline $1 \mathrm{~s}$ & $\mathrm{~A}$ & & & $\mathrm{~A}$ & & & $\mathrm{~A}$ & $\mathrm{~A}$ \\
\hline \multicolumn{9}{|l|}{$2 a$} \\
\hline $2 \mathrm{~b}$ & $\mathrm{~A}$ & & $\mathrm{~A}$ & & & & $\mathrm{~A}$ & $\mathrm{~A}$ \\
\hline $2 c$ & & $\mathrm{~A}$ & & & & & 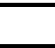 & \\
\hline \multicolumn{9}{|l|}{$2 \mathrm{~d}$} \\
\hline $2 \mathrm{e}$ & & & & & $\mathrm{A}$ & $\mathrm{A}$ & $\mathrm{A}$ & $\mathrm{A}$ \\
\hline $2 \mathrm{f}$ & & $\mathrm{A}$ & & & & & $\mathrm{A}$ & $\mathrm{A}$ \\
\hline $2 \mathrm{~g}$ & & & $\mathrm{~A}$ & & & $\mathrm{~A}$ & & \\
\hline \multicolumn{9}{|l|}{$2 \mathrm{~h}$} \\
\hline \multicolumn{9}{|l|}{$2 \mathrm{i}$} \\
\hline $2 j$ & $\mathrm{~A}$ & & $\mathrm{~A}$ & $\mathrm{~A}$ & $\mathrm{~A}$ & & $\mathrm{~A}$ & $\mathrm{~A}$ \\
\hline $3 a$ & & & & & & $\mathrm{~A}$ & $\mathrm{~A}$ & \\
\hline $3 b$ & & & $\mathrm{~A}$ & $\mathrm{~A}$ & $\mathrm{~A}$ & & & \\
\hline $3 c$ & $\mathrm{~A}$ & & $\mathrm{~A}$ & $\mathrm{~A}$ & $\mathrm{~A}$ & & $\mathrm{~A}$ & $\mathrm{~A}$ \\
\hline $3 \mathrm{~d}$ & & & $\mathrm{~A}$ & & $\mathrm{~A}$ & & $\mathrm{~A}$ & $\mathrm{~A}$ \\
\hline $3 e$ & & & & & & $\mathrm{~A}$ & & \\
\hline \multicolumn{9}{|l|}{$3 f$} \\
\hline \multicolumn{9}{|l|}{$3 g$} \\
\hline $3 \mathrm{~h}$ & & & & & & $\mathrm{~A}$ & & \\
\hline $3 \mathrm{i}$ & & $\mathrm{A}$ & & & & & & $\mathrm{A}$ \\
\hline \multicolumn{9}{|l|}{$3 j$} \\
\hline \multicolumn{9}{|l|}{$3 \mathrm{k}$} \\
\hline 31 & $\mathrm{~A}$ & & $\mathrm{~A}$ & & $\mathrm{~A}$ & & & $\mathrm{~A}$ \\
\hline $3 \mathrm{~m}$ & & & & & & $\mathrm{~A}$ & & \\
\hline $4 a$ & A & & & $\mathrm{A}$ & $\mathrm{A}$ & A & & \\
\hline $4 \mathrm{~b}$ & & & & & & & & $\bar{A}$ \\
\hline $4 c$ & $\mathrm{~A}$ & $\mathrm{~A}$ & & & $\mathrm{~A}$ & & $\mathrm{~A}$ & \\
\hline $4 d$ & & & & & & $\mathrm{~A}$ & $\mathrm{~A}$ & \\
\hline $4 \mathrm{e}$ & $\mathrm{A}$ & $\mathrm{A}$ & $\mathrm{A}$ & & $\mathrm{A}$ & & $\mathrm{A}$ & $\mathrm{A}$ \\
\hline $4 \mathrm{f}$ & $\mathrm{A}$ & 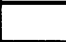 & $\mathrm{A}$ & & & . & $\mathrm{A}$ & \\
\hline
\end{tabular}

$\mathbf{A}=$ skill assessed

Figure 3 Many skills may be developed through the Case Studies, and each Case Study contains a Skills Check to enable teachers to monitor both the skills that are developed as well as those that are assessed by each task. This table provides an overview only of those skills that are being assessed within each of the Case Studies 